\title{
Unifying CP violations of quark and lepton sectors
}

\author{
Jihn E. Kim ${ }^{1,2,3, a}$, Soonkeon Nam ${ }^{1}$ \\ ${ }^{1}$ Department of Physics, Kyung Hee University, 26 Gyungheedaero, Dongdaemun-Gu, Seoul 02447, Republic of Korea \\ ${ }^{2}$ Center for Axion and Precision Physics Research (IBS), 291 Daehakro, Yuseong-Gu, Taejon 34141, Republic of Korea \\ ${ }^{3}$ Department of Physics, Seoul National University, 1 Gwanakro, Gwanak-Gu, Seoul 08826, Republic of Korea
}

Received: 25 August 2015 / Accepted: 23 November 2015 / Published online: 8 January 2016

(C) The Author(s) 2015. This article is published with open access at Springerlink.com

\begin{abstract}
A preliminary determination of the Dirac phase in the PMNS matrix is $\delta_{\mathrm{PMNS}} \approx-\frac{\pi}{2}$. A rather accurately determined Jarlskog invariant $J$ in the CKM matrix is close to the maximum. Since the phases in the CKM and PMNS matrices will be accurately determined in the future, it is an interesting problem to relate these two phases. This can be achieved in a families-unified grand unification if the weak $\mathrm{CP}$ violation is introduced spontaneously $\grave{a}$ la Froggatt and Nielsen at a high energy scale, where only one meaningful Dirac CP phase appears.
\end{abstract}

\section{Introduction}

At present, the real angles of the Cabibbo-KobayashiMaskawa (CKM) matrix are rather accurately determined [1], which makes it possible to pin down the invariant phase $\delta_{\mathrm{CKM}}$ into three possibilities $\alpha, \beta$, and $\gamma$ of the unitarity triangle [2]. The physically observable $\mathrm{CP}$ magnitude is the Jarlskog determinant $J[3,4]$ which can be expressed as $J=$ (real angles) $\cdot \sin \delta_{\mathrm{CKM}}$. Depending on the parametrization, $\delta_{\mathrm{CKM}}$ can be $\alpha, \beta$, or $\gamma$. The maximality of $J$ is a different concept from the maximality of the phase $\delta_{\mathrm{CKM}}$. The maximality of phase is $\delta_{\mathrm{CKM}} \simeq \pm \frac{\pi}{2}$. Even though $\delta_{\mathrm{CKM}} \neq \pm \frac{\pi}{2}$, $J$ can be maximal in the vicinity of a given $\delta_{\mathrm{CKM}}$, which can be checked by varying the real angles together with $\delta_{\mathrm{CKM}}$ within the experimentally allowed bounds [2]. $\delta_{\mathrm{CKM}}$ is close to the maximum $90^{\circ}$ in the parametrization suggested by Kim and Seo (KS) [5,6] and Kobayashi and Maskawa (KM) [2,7]. The particle data group (PDG) compilation of the invariant phase is $\alpha=\left(85.4_{-3.8}^{+3.9}\right)^{\circ}$ [8]. Thus, $\delta_{\mathrm{CKM}}=\alpha$ in the $\mathrm{KS}$ and $\mathrm{KM}$ parametrizations shows that $J$ is close to maximum. The same maximality of $J$ is also drawn from the Chau-Keung (CK) parametrization where $\delta_{\mathrm{CKM}}=\gamma$ [2].

In this paper, we use the KS parametrization as an explicit example [6] where the only complex number in the CKM

a e-mail: jihnekim@gmail.com matrix [7,9] is the invariant Jarlskog phase itself $[3,4]$. Here, the phase is multiplied to the ( $\left.\operatorname{small} \mathrm{O}\left(\left|\lambda^{3}\right|\right)\right)$ whole element, $V_{\mathrm{CKM}(31)}$, which makes it possible to appreciate the weak $\mathrm{CP}$ violation from $V_{\mathrm{CKM}}$ itself. For the Pontecorvo-MakiNakagawa-Sakada (PMNS) matrix [10,11], we have already entered into an era of determining a Dirac phase $\delta_{\text {PMNS }}$ modified by some Majorana phases, with a preliminary result close to $\mp 90^{\circ}$ at a $2 \sigma$ level $[12,13]$. Therefore, it is timely to ask a question now whether one can relate $\delta_{\mathrm{PMNS}}$ and $\delta_{\mathrm{CKM}}$ or not. To relate the CKM and PMNS phases, one can consider using a grand unified theory (GUT) which unifies quarks and leptons with a suitable scheme unifying families $[14,15]$.

Most family unification models assume a factor group $G_{f}$ in addition to the Standard Model (SM) or GUT, where continuous symmetries such as $\mathrm{SU}(2)$ [16], $\mathrm{SU}(3)$ [17-19], or $\mathrm{U}(1)$ 's [20,21], and discrete symmetries such as $S_{3}$ [22-24], $A_{4}[25,26], \Delta_{96}$ [27], $\mathbf{Z}_{12}[28,29]$ for $G_{f}$ have been considered. A full unification of GUT families in the sense that the couplings of the family group are unified with the three gauge couplings of the SM is by unifying the families in a simple gauge group based on $\mathrm{SU}(N)$ [14]. Along this line, one of the authors has recently suggested a families-unification based on an anti-SU(7) GUT, SU(7) $\times U(1)$ [15], which in fact unifies family couplings with three gauge couplings of the SM. The string derived anti-SU(7) [15] has a merit in that it is free from gauge anomalies and from the gravity spoiling of some discrete symmetries [30-37]. In this paper, however, we discuss this at the field theory level of the SM.

We need a true unification of GUT families. Even that requirement is used only when we argue for the possibility of $\delta_{\mathrm{CKM}} \simeq \pm \delta_{\text {PMNS }}$ based on the assumption that the Dirac phases in the CKM and PMNS matrices originates from the spontaneous CP violation mechanism [38] at a high energy scale à la Froggatt and Nielsen [20].

If one allows completely general complex Yukawa couplings in the quark and lepton sectors, one cannot relate $\delta_{\text {PMNS }}$ and $\delta_{\text {CKM }}$. If all Yukawa couplings are real, the weak 
$\mathrm{CP}$ violation must be introduced by non-removable complex vacuum expectation values (VEVs). To have a relation without any other parameters, such as in the relation $\delta_{\mathrm{PMNS}}= \pm \delta_{\mathrm{CKM}}$, only one phase must be introduced in the whole theory such as in the unification of GUT families. To mimic the KM weak $\mathrm{CP}$ at low energy, the complex VEV must be that of a SM singlet $[39,40]$ as performed in [41].

In Sect. 2, we define the CKM and PMNS matrices. Section 3 is the main part of the paper, where the diagonalization of mass matrices and the parametrization of the CKM and PMNS matrices are discussed. In Sect. 4, we present the diagonalization mechanisms of $M^{(u)}$ and $M^{(v)}$, needed for relating $\delta_{\mathrm{CKM}}$ and $\delta_{\mathrm{PMNS}}$, and we present a relation in the anti-SU(7) model. Section 5 is for a conclusion.

\section{The CKM and PMNS matrices}

In this short section, we define the CKM and PMNS matrices. Let the quark and lepton representations of the SM be

$$
\begin{gathered}
q_{a L}=\left(\begin{array}{c}
u_{a} \\
d_{a}
\end{array}\right)_{L}, u_{a L}^{c}, d_{a L}^{c} ; \ell_{a L}=\left(\begin{array}{c}
v_{a} \\
e_{a}
\end{array}\right)_{L}, e_{a L}^{+}, \\
N_{a L}, a=1,2,3,
\end{gathered}
$$

where $a$ is the family indices in the weak eigenstates. The $(i j)$ element of the CKM matrix is defined as the $W_{\mu}^{+}$boson coupling to the current $\bar{u}_{L}^{\text {(mass } i)} \gamma^{\mu} d_{L}^{\text {(mass } j)} W_{\mu}^{+}$where $u^{\text {(mass } i)}$ and $d^{(\text {mass } i)}$ are the mass eigenstates,

$$
\begin{aligned}
& u^{(\operatorname{mass} 1)}=u, u^{(\operatorname{mass} 2)}=c, u^{(\operatorname{mass} 3)}=t, \\
& d^{(\operatorname{mass} 1)}=d, d^{(\operatorname{mass} 2)}=s, d^{(\operatorname{mass} 3)}=b .
\end{aligned}
$$

Choosing the mass eigenstate $d$ quarks is quite general since this step is considered after diagonalizing the $d$-type quark masses, and below we will not touch upon the redefinition possibility of $d$-type quarks. This choice is useful in the flipped-SU(5) GUT [42,43] from string origin [44,45], where $Q_{\text {em }}=-\frac{1}{3}$ quarks and heavy neutrino $N$ 's are grouped in 10, of which we do not intend to address the question how they couple. Namely, we intend to avoid as much as possible the heavy neutrino sector in our discussions. Then the $(i j)$ element is the matrix element diagonalizing the weak states $u_{L}^{(\operatorname{mass} i)}=\sum_{a} V_{i a} u_{L}^{a}$,

$V_{i j}^{\mathrm{CKM}}=V_{i a}$.

This is depicted in Fig. 1a. In the standard presentation of the KM model, for the $W_{\mu}^{+}$coupling we consider only the unitary matrix diagonalizing the $q_{L}^{a}$ fields, together with the up-type and down-type quark phases. In our case, we already diagonalized down-type quarks and we consider the phases of right-handed up-type quarks instead of the phases of lefthanded down-type quarks. We draw the intermediate line

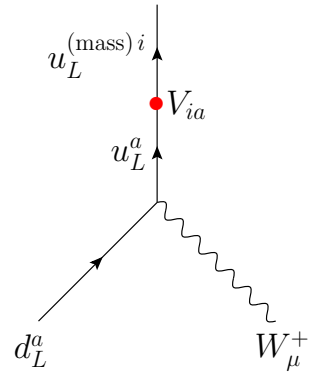

(a)

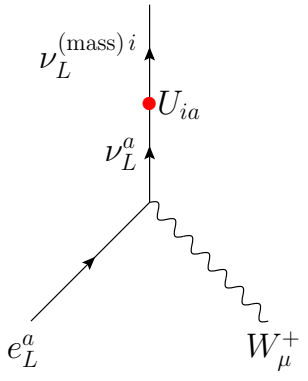

(b)
Fig. 1 The charged currents defining the CKM and PMNS matrices: a quarks, and $\mathbf{b}$ leptons. In $\mathbf{a}$, to show the unitary transformation explicitly we represent the coupling $g V_{i a}$ as the red bullet and $u_{L}^{a}$ line, and similarly in b

$u_{L}^{a}$ in Fig. 1a to imply that it is related to $u_{R}^{b}$ in the mass diagonalization process.

Similarly, we define the $W_{\mu}^{+}$coupling to the lepton currents, $\bar{v}_{L}^{\text {(mass } i)} \gamma^{\mu} e_{L}^{(\text {mass } j)} W_{\mu}^{+}$, where $v^{(\text {mass } i)}$ and $e^{(\text {mass } i)}$ are the mass eigenstates. These leptonic currents define the PMNS matrix. There will be only three phase freedoms of neutrinos since we will not touch upon the charged leptons. There is another reason to use the bases where charged leptons are mass eigenstates. It is because the masses of $e, \mu$, and $\tau$ are known accurately. Then the PMNS matrix is

$U_{i j}^{\mathrm{PMNS}}=U_{i a}$.

Namely, the matrix diagonalizing the SM neutrinos is $U_{i a}$,

$v_{L}^{(\operatorname{mass} i)}=\sum_{a} U_{i a} v_{L}^{a}$

and the PMNS matrix is depicted in Fig. $1 \mathrm{~b}$.

\section{Yukawa couplings, masses, and spontaneous CP violation}

Not to allow some complication on the flavor changing neutral current (FCNC) issue, let us introduce only one pair of BEH doublets: $H_{d}$ coupling to $d$-type quarks and $H_{u}$ coupling to $u$-type quarks, probably by a Peccei-Quinn symmetry $[46,47]$. The masses for the charged leptons and $Q_{\mathrm{em}}=-\frac{1}{3}$ quarks are arising from the Dirac Yukawa couplings $f_{a b}^{(e)} \ell_{a} e_{b}^{+} H_{d}$ and $f_{a b}^{(d)} q_{a} d_{b}^{c} H_{d}$, respectively. Let us assume $f_{a b}^{(e, d)}$ and the $\operatorname{VEV}\left\langle H_{d}^{0}\right\rangle$ to have real values, and diagonalize the charged lepton and $Q_{\mathrm{em}}=-\frac{1}{3}$ quark mass matrices, without affecting the $\mathrm{CP}$ phase we would like to introduce.

One may encounter a situation where the Higgs field coupling to $d$-type quarks develop complex VEVs, in which case the equations take a bit more complicated forms. Not to clutter to this situation, we do not choose this vacuum. 
Then all CP violation effects are assumed to arise from the Yukawa couplings of $H_{u}$,

$$
\begin{aligned}
& f_{a b}^{(u)} q_{a} u_{b}^{c} H_{u}, \\
& f_{a b}^{(v)} \ell_{a} N_{b} H_{u} .
\end{aligned}
$$

Let us assume that $f_{a b}^{(u)}$ and $f_{a b}^{(v)}$ are real. Then one $H_{u^{-}}$ type doublet cannot introduce a weak $\mathrm{CP}$ violation spontaneously [38] even if it develops a complex VEV, say $\left\langle H_{u}^{0}\right\rangle=v_{u} e^{i \delta}$, since the phase appears as an overall one in the up-type quark mass matrix. Therefore, we introduce phases in $f_{a b}^{(u)}$ and $f_{a b}^{(v)}$ at a high energy scale by a complex VEV of one SM singlet field $X$ à la Froggatt and Nielsen [20]. Here, we allow $X$ couplings, not only one power but many different powers of $X$. Effectively, it amounts to introducing many $H_{u}$ 's, but the FCNCs are suppressed by superheavy masses of $X$.

By some symmetry structure of the theory, the $X$ coupling can be made flavor-dependent $[48,49]$. Now, let us proceed in this scheme to relate $\delta_{\mathrm{CKM}}$ and $\delta_{\mathrm{PMNS}}$.

The Dirac Yukawa coupling (6) gives masses to both $Q_{\mathrm{em}}=\frac{2}{3}$ quarks and neutrinos. Let us take $\left\langle H_{u}\right\rangle$ real. If it were complex, its phase can be removed by redefining the $Q_{\mathrm{em}}=\frac{2}{3}$ quark fields. Then, starting from the weak eigenstate bases, we obtain

$$
\begin{aligned}
L^{(u)} & =\bar{u}_{R}^{b} f_{a b}^{(u)}\left\langle H_{u}^{0}\right\rangle u_{L}^{a}+\bar{u}_{R}^{b} f_{b a}^{(u) *}\left\langle H_{u}^{0}\right\rangle u_{L}^{a} \\
& =v_{u} \bar{u}_{R}^{b} f_{a b}^{(u)}\left(V^{\dagger}\right)_{a i} u_{L}^{(\operatorname{mass} i)}+v_{u} \bar{u}_{R}^{b} f_{b a}^{(u) *}\left(V^{\dagger}\right)_{a i} u_{L}^{(\operatorname{mass} i)}
\end{aligned}
$$

and

$$
L^{(\nu)}=\bar{N}_{R}^{b} f_{a b}^{(v) *}\left\langle\tilde{H}_{u}^{0}\right\rangle v_{L}^{a}=v_{u} \bar{N}_{R}^{b} f_{a b}^{(v) *}\left(U^{\dagger}\right)_{a i} v_{L}^{(\operatorname{mass} i)}
$$

where $\tilde{H}_{u}=i \sigma_{2} H_{u}^{*}=\left(H_{u}^{0 *},-H^{-}\right)^{T}$ with $\left\langle H_{u}^{0}\right\rangle=$ $\left\langle\tilde{H}_{u}^{0 *}\right\rangle=v_{u}$. In Eq. (8), the fact of there being only one chirality, say the left-handedness of the SM neutrinos, is used. As commented on above, the Yukawa couplings $f_{a b}^{(u)}$ and $f_{a b}^{(v)}$ can be complex à la Froggatt and Nielsen. In Fig. 2, we visualize the mass terms of the up-type quarks and neutrinos. For the neutrinos, the Type-I seesaw mechanism is used.

To relate the phases in the CKM and PMNS matrices, the phases of $f_{a b}^{(u)}$ and $f_{a b}^{(v)}$ must be related. Here, we need some model for the family unification. As commented on in the Introduction, we use the top-down approach, i.e. the model of the true unification of GUT families based on anti$\mathrm{SU}(7)$ from string compactification. In the family unification models from the bottom-up approach, one has to check the vanishing of some anomalous terms via the discrete gauge symmetry [30], which is not a simple task.

The 16 chiral fields of the SM are grouped into $\mathbf{1 0}, \overline{\mathbf{5}}$ and $\mathbf{1}$ of the flipped-SU(5) spectrum (or anti-SU(5) [43]), contained in anti-SU(7) [15], thus:

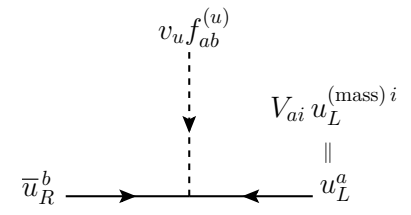

(a)

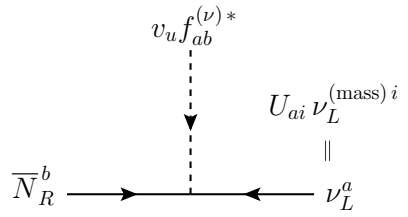

(b)

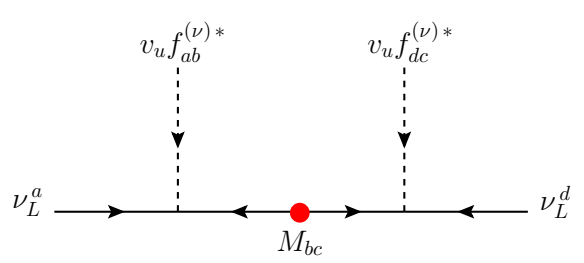

(c)

Fig. 2 The diagrams for masses of up-type quarks and neutrinos: a the Dirac mass of $u^{a}, \mathbf{b}$ the Dirac mass of $N$ and $\nu$, and $\mathbf{c}$ the seesaw mass of the SM neutrinos. The bullet in $\mathbf{c}$ is the Majorana mass $M_{b c}$ of the heavy neutrinos $N^{b}$ and $N^{c}$

$\mathbf{1 0}_{a}=\left(\begin{array}{ccc} & \left|u_{a}\right| \\ d_{a}^{c} & \mid & \mid N_{a} \\ & \left|d_{a}\right|\end{array}\right)_{L}, \overline{\mathbf{5}}_{a}=\left(\begin{array}{c}u_{a}^{c} \\ -- \\ \ell_{a}\end{array}\right)_{L}, \mathbf{1}_{a}=e_{a L}^{+}$,

where bars separate different color representations. Here, $\ell_{a}$ is the $a$ th lepton doublet,

$\ell_{a}=\left(\begin{array}{c}v_{a} \\ e_{a}\end{array}\right)$.

Then the couplings in Eqs. (7) and (8) are the same, $F_{a b}=$ $f_{a b}^{(u)}=f_{a b}^{(v)}$. The relevant phases are read for the same order of family indices $f_{a b}$ 's in Eqs. (7) and (8). Thus, the phases in the quark and lepton charged current are made to contain $\delta$. The argument is presented in Sect. 4 .

The physically relevant quantity measurable experimentally is the Jarlskog determinant $J$. The quark sector $J_{\text {CKM }}$ contains the Jarlskog phase $\delta_{\mathrm{CKM}}$ and the lepton sector $J_{\text {PMNS }}$ contains the Jarlskog phase $\delta_{\text {PMNS }}$. Here, we suggest how these are related to $\delta$. The Jarlskog determinant $J$ can be expressed simply as $J=\operatorname{Im} V_{31}^{*} V_{22}^{*} V_{13}^{*}$ [5]. It has the form $J=$ (product of real CKM angles) . $\sin \delta_{\mathrm{CKM}}=\operatorname{Im}\left[\right.$ (product of real CKM angles) $\left.e^{i \delta_{\mathrm{CKM}}}\right]$. The invariant Jarlskog phase $\delta_{\mathrm{CKM}}$ is determined up to three classes, $\alpha, \beta$ and $\gamma$ of PDG, depending on the parametrization schemes [2]. Let us use the simple KS form for the CKM matrix [6]

$$
\begin{aligned}
& V_{\mathrm{CKM}}^{\mathrm{KS}} \\
& =\left(\begin{array}{ccc}
c_{1} & s_{1} c_{3} & s_{1} s_{3} \\
-c_{2} s_{1} & e^{-i \delta_{\mathrm{CKM}}} s_{2} s_{3}+c_{1} c_{2} c_{3} & -e^{-i \delta \delta_{\mathrm{CKM}} s_{2} c_{3}}+c_{1} c_{2} s_{3} \\
-e^{i \delta_{\mathrm{CKM}}} s_{1} s_{2} & -c_{2} s_{3}+c_{1} s_{2} c_{3} e^{i \delta_{\mathrm{CKM}}} & c_{2} c_{3}+c_{1} s_{2} s_{3} e^{i \delta_{\mathrm{CKM}}}
\end{array}\right)
\end{aligned}
$$

where the real CKM angles are $c_{i}=\cos \theta_{i}, s_{i}=\sin \theta_{i}$ for $i=1,2,3$. One merit of the form (10) is that $e^{i \delta_{\text {СКM }} \text { is the }}$ 
overall phase in the small element, i.e. in the (31) element in $V_{\mathrm{CKM}}^{\mathrm{KS}}$. This makes it easy to glimpse the magnitude of the Jarlskog determinant. In comparison, note that $e^{i \delta_{\text {СKM }} \text { does }}$ not appear as an overall phase in the CK parametrization.

Similarly, the KS form for the PMNS matrix can be written from Eq. (10) by replacing $\theta_{i} \rightarrow \Theta_{i}, s_{i} \rightarrow S_{i}, c_{i} \rightarrow C_{i}$, and $\delta_{\mathrm{CKM}} \rightarrow \delta_{\mathrm{PMNS}}$. Of course, the real angles $\theta_{i}$ and $\Theta_{i}$ are not identical, because the mass matrices to be diagonalized involve an unrelated ingredient. We note that

$$
\begin{aligned}
J_{\mathrm{CKM}} & =-\operatorname{Im}\left[V_{31} V_{22} V_{13}\right]=c_{1} c_{2} c_{3} s_{1}^{2} s_{2} s_{3} \sin \delta_{\mathrm{CKM}}, \\
J_{\mathrm{PMNS}} & =-\operatorname{Im}\left[U_{31} U_{22} U_{13}\right]=C_{1} C_{2} C_{3} S_{1}^{2} S_{2} S_{3} \sin \delta_{\mathrm{PMNS}} .
\end{aligned}
$$

For the neutrino masses, the heavy Majorana couplings are involved in addition. The latter couplings lead to large values of $\left|S_{i}\right|$, in contrast to small values of $\left|s_{i}\right|$. The Majorana couplings can introduce two more Majorana phases. These two Majorana phases $\delta_{M}$ cannot be measured independently from the Dirac phase in the laboratory experiments. Out of the nine parameters of $U$, three neutrino phases can be used to reduce to six. To make the determinant real, two Majorana phases (usually written as two diagonal phases) are removed to write $J_{\text {PMNS }}$. A newly defined Dirac phase in the PMNS matrix is a combination from $\operatorname{VEV}\langle X\rangle=V e^{i \delta}$ and $e^{-i \delta_{M}}$ of $M_{N}^{-1}$ of Fig. 2 . In this way, the phases $\delta_{\mathrm{CKM}}$ and $\delta_{\mathrm{PMNS}}$ can be related. We assumed that the phase of $\langle X\rangle$ is the only source of $\mathrm{CP}$ violation, including the heavy neutrino sector. The mass matrix of $m_{a b}$ of Fig. 2 is a combination of phases of $f_{a b}^{\mathcal{*}}$ and the phase $e^{-i \delta_{M} / 2}$, which must define $\delta_{\mathrm{PMNS}}$. If $\delta$ of $\operatorname{VEV}\langle X\rangle$ is zero, there is no $\mathrm{CP}$ violation in the quark sector, and also in the lepton sector, i.e. $\delta_{\mathrm{CKM}}=0, \delta_{\mathrm{PMNS}}=0$, and $\delta_{M}=0$ in the full theory. We present a physical argument to glimpse the situation without a detailed study. The Jarlskog triangles in Fig. 3 become lines and $J=0$ if $\delta_{\mathrm{CKM}}$ and $\delta_{\mathrm{PMNS}}$ are integer multiples of $\pi$. Then there is no physically measurable CP violation. Whatever happens in the calculation, this must be the case. There will be no weak CP violation if $\delta$ (the phase of $X$ ) has integer multiples of $\pi$ since the VEV of $X$ does not introduce an imaginary component in the whole theory. Therefore, $\delta_{\mathrm{CKM}}$ and $\delta_{\mathrm{PMNS}}$ must be integer multiples of $\delta$ not to introduce $\mathrm{CP}$ violation in the case $\delta=\pi$. This must be true even if we consider the heavy neutrinos since the heavy neutrinos belong to a part in the theory. We have

$\delta_{\mathrm{CKM}}=n_{1} \delta, \delta_{\mathrm{PMNS}}=n_{2} \delta$.

Because our argument on the vanishing of $J$ does not depend on the sign of $\delta_{\mathrm{CKM}}$, we can take both signs for $\delta_{\mathrm{CKM}}$ and $\delta_{\mathrm{PMNS}}$, and for $n_{1}=n_{2}$ we have

$\delta_{\mathrm{PMNS}}= \pm \delta_{\mathrm{CKM}}$.

Note that we obtained this result by assuming that only the phase of $\langle X\rangle$ is the source of the weak CP violations, includ-

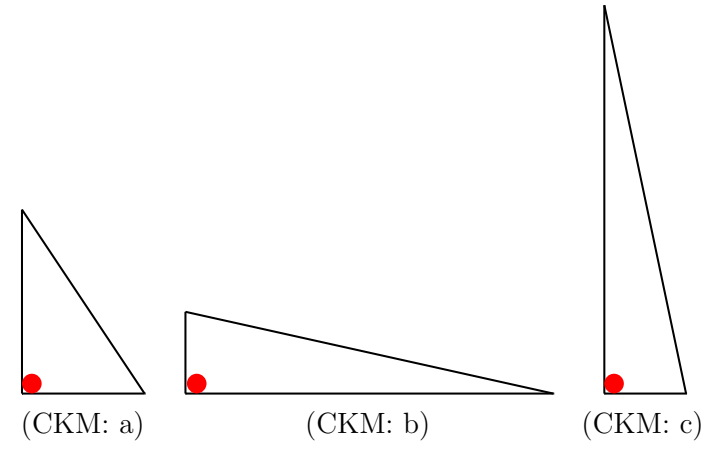

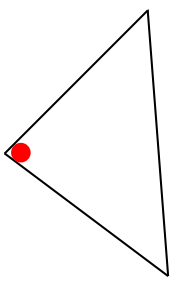

(PMNS: a)

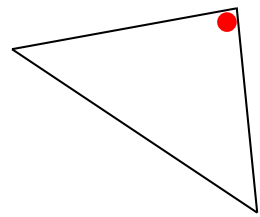

(PMNS: b)

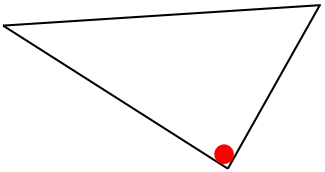

(PMNS: c)
Fig. 3 Schematic shapes of $J$ for the CKM and PMNS triangles. All of them have one angle with $\delta_{\mathrm{CKM}}= \pm \delta_{\mathrm{PMNS}}$ in our scenario

ing the heavy neutrino sector. Namely, in the full theory the Majorana phases must also arise without coupling to $X$ or by the phase of $\langle X\rangle$. This physical argument does not depend on which parametrization we use for the PMNS matrix. Namely, we can use any parametrization for the PMNS matrix as far as $\delta$ PMNS is one of $\alpha, \beta$, and $\gamma$ of PDG. ${ }^{1}$ In other words, an accurate determination of $\delta$ PMNS, which will be the common angle appearing in all six leptonic Jarlskog triangles, will fix $\delta_{\mathrm{CKM}}$ and lead to a choice of one class from the CKM matrices. $^{2}$ In Fig. 3, we show that one Jarlskog phase appears in the CKM and PMNS triangles if the assumptions on our $\mathrm{CP}$ violation are satisfied.

We followed the flipped-SU(5) language so far. We can present the same line of reasoning line by line for the GeorgiGlashow model also [50]: all Yukawa couplings are real and the VEVs $\left\langle H_{u}\right\rangle$ and $\left\langle H_{d}\right\rangle$ are real and $\langle X\rangle$ obtaining a complex VEV couples only to charged leptons and $Q_{\mathrm{em}}=-\frac{1}{3}$ quarks. In this case, the GUT breaking is by a VEV of an adjoint BEH field $\langle\mathbf{2 4}\rangle$. However, a dilemma here is the difficulty of obtaining the SU(5) adjoint BEH field from string compactification [51]. In addition, the doublet-triplet splitting with the adjoint BEH field needs a fine tuning between

\footnotetext{
1 We assume the unification of CP phases and the CKM phase can be one of $\alpha, \beta$, and $\gamma$, which are already determined by the $\mathrm{O}\left(\lambda^{3}\right)$ unitarity triangle.

${ }^{2}$ Note that $\delta_{\mathrm{CKM}}$ is also the common angle in all six quark Jarlskog triangles, but in each triangle except the one with angles $\alpha, \beta$ and $\gamma$ in the PDG book one side is always small, which makes it difficult to measure the angles at the end of the small side.
} 
the $\operatorname{VEV}\langle\mathbf{2 4}\rangle$ and a free mass parameter for the adjoint scalar $m^{2} \mathbf{2 4}^{2}$.

\section{Relating the phase of $\langle X\rangle$ to $\delta_{\mathrm{CKM}}$ and $\delta_{\mathrm{PMNS}}$}

Even though the physical argument presented in the previous section is enough for relating $\delta_{\mathrm{PMNS}}$ and $\delta_{\mathrm{CKM}}$, here we present a scheme in detail of how they are connected. The common phase in $\langle X\rangle=V e^{i \delta}$ will appear in the $(i j)$ element of the quark or lepton mass matrix with the form $A_{i j}\left(e^{i \delta}\right)^{n}$ if the singlet $X^{n}$ is located in the $(i j)$ element. We work in the bases where $M^{(d)}$ and $M^{(e)}$ are already diagonalized, and the left-hand unitary matrices diagonalizing $M^{(u)}$ and $M^{(v)}$ are the CKM and PMNS matrices, respectively.

The parameters of mass matrix $M^{(u)}$ combine to produce the phase in the CKM matrix. For a complex mass matrix $M^{(u)}$, there are 18 real parameters. It can be diagonalized by a bi-unitary transformation by $U^{L}$ and $U^{R}$. Thus, there are 36 parameters to be considered initially from $M^{(u)}, U^{L}$, and $U^{R}$. The left- and right-hand sides of the diagonalization relation, $U^{L \dagger} M^{(u)} U^{R}=e^{i \alpha}$ (real diagonal mass matrix), have the same overall phase, corresponding to the baryon number conservation. Disregarding the baryon number, by making $\operatorname{Det} M^{(u)}$ real, so that the diagonalized masses are real, we consider 35 real parameters in the diagonalizing conditions. The condition $U^{L \dagger} M^{(u)} U^{R}=$ (real diagonal mass matrix) gives 18 relations. With these conditions imposed, there remain 17 independent parameters from 35 . Out of 17 , five (since the overall phase cannot be used) can be removed by redefining L- and R-handed $u$-type quark phases. Thus, there remain 12 independent parameters. Out of 12, 11 parameters remain as physical ones, three real $u$-type quark masses, four angles of $U^{L}$, and four angles of $U^{R}$. But the parameters in $U^{R}$ are hidden at low energy. ${ }^{3}$ Now, there is one more (phase) parameter remaining. So, we must use one more relation to fix the theory completely. It is the relation $\delta_{\mathrm{CKM}}=n_{1} \delta$.

Let us parametrize $U^{L, R}$ as given in (10) in which case there is no more freedom to rotate the quark fields,

$$
\begin{aligned}
M_{\alpha \beta} & =U_{i \alpha}^{L *} M_{i j}^{(u)} U_{j \beta}^{R} \text {, i.e. } M_{1 \beta}=U_{i 1}^{L *} M_{i j}^{(u)} U_{j \beta}^{R} \\
& =U_{i 1}^{L *} M_{i 1}^{(u)} U_{13}^{R}+U_{i 1}^{L *} M_{i 2}^{(u)} U_{23}^{R}+U_{i 1}^{L *} M_{i 3}^{(u)} U_{33}^{R} .
\end{aligned}
$$

In (10), we try to fix the phase $\delta_{\mathrm{CKM}}$ from the (31) element since there is no ambiguity in choosing the phase because it is an overall one, $\propto e^{i \delta_{\mathrm{CKM}}}$. From (14), note that the (31) element appears only in $M_{1 \beta}$ : in the factor $U_{i 1}^{L *}$. In the other elements, they are real or a phase does not appear as an overall one in Eq. (10). We have

\footnotetext{
${ }^{3}$ We choose the same number of parameters for $U^{R}$ as for $U^{L}$, since the

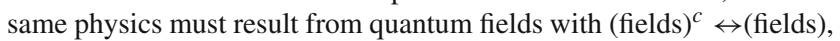
which is equivalent to $L \leftrightarrow R$.
}

$$
\begin{aligned}
M_{13}= & U_{11}^{L *} M_{11}^{(u)} U_{13}^{R}+U_{21}^{L *} M_{21}^{(u)} U_{13}^{R}+\underline{U_{31}^{L *} M_{31}^{(u)}} U_{13}^{R} \\
& +U_{11}^{L *} M_{12}^{(u)} U_{23}^{R}+U_{21}^{L *} M_{22}^{(u)} U_{23}^{R}+U_{31}^{L *} M_{32}^{(u)} U_{23}^{R} \\
& +U_{11}^{L *} M_{13}^{(u)} U_{33}^{R}+U_{21}^{L *} M_{23}^{(u)} U_{33}^{R}+U_{31}^{L *} M_{33}^{(u)} U_{33}^{R}=0,
\end{aligned}
$$

$$
\begin{aligned}
M_{12}= & U_{11}^{L *} M_{11}^{(u)} U_{12}^{R}+U_{21}^{L *} M_{21}^{(u)} U_{12}^{R}+\underline{U_{31}^{L *} M_{31}^{(u)}} U_{12}^{R} \\
& +U_{11}^{L *} M_{12}^{(u)} U_{22}^{R}+U_{21}^{L *} M_{22}^{(u)} U_{22}^{R}+U_{31}^{L *} M_{32}^{(u)} U_{22}^{R} \\
& +U_{11}^{L *} M_{13}^{(u)} U_{32}^{R}+U_{21}^{L *} M_{23}^{(u)} U_{32}^{R}+U_{31}^{L *} M_{33}^{(u)} U_{32}^{R}=0
\end{aligned}
$$

$$
\begin{aligned}
M_{11}= & U_{11}^{L *} M_{11}^{(u)} U_{11}^{R}+U_{21}^{L *} M_{21}^{(u)} U_{11}^{R}+\underline{U_{31}^{L *} M_{31}^{(u)}} U_{11}^{R} \\
& +U_{11}^{L *} M_{12}^{(u)} U_{21}^{R}+U_{21}^{L *} M_{22}^{(u)} U_{21}^{R}+U_{31}^{L *} M_{32}^{(u)} U_{21}^{R} \\
& +U_{11}^{L *} M_{13}^{(u)} U_{31}^{R}+U_{21}^{L *} M_{23}^{(u)} U_{31}^{R}+U_{31}^{L *} M_{33}^{(u)} U_{31}^{R}=m_{u} .
\end{aligned}
$$

Since $U_{1 \alpha}^{R}$ is real in the KS form, we choose $\delta_{\mathrm{CKM}}$ as the argument of $M_{31}^{(u)}$, making the underlined parts of Eqs. (15), (16), and (17) real. The number of conditions in $M_{\alpha \beta}=$ $U_{i \alpha}^{L *} M_{i j}^{(u)} U_{j \beta}^{R}$ is 18 , which we counted before. We make this number 19 by imposing an extra condition, $U_{31}^{L *} M_{31}^{(u)}=$ real, where $M_{31}^{(u)} \propto\langle X\rangle \propto e^{i \alpha}$. This is a detailed construction of $\delta_{\mathrm{CKM}}$ from $M^{(u)}$.

In the leptonic sector, consider Fig. 2. We assumed that the charged lepton mass matrix $M^{(e)}$ is already diagonalized. The symmetric neutrino mass term is $\frac{1}{2} v^{T} M^{(v)} v$, violating the lepton number by two units. Here, $M^{(v)}$ is complex and symmetric. A complex symmetric matrix $A$ can be 'diagonalized' using one unitary matrix $U$, where $U^{T} A U$ is a real diagonal matrix, which is called the Autonne-Takagi factorization [52,53]. It is not a unitary transformation,

$$
M_{\alpha \beta}^{(\nu) \text { diag. }}=U_{i \alpha} M_{i j}^{(v)} U_{j \beta}
$$

Even though the theory breaks the lepton number, the overall phase cannot be used in the diagonalizing condition (18), since both in the left- and right-hand sides the lepton number is broken by the same unit. Thus, the independent number of conditions in (18) is 17. The (31) elements appear

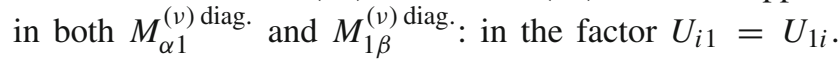
In the other elements, they are real or a phase does not appear as an overall one in Eq. (10). Note that $U_{31}$ appears in

$$
\begin{aligned}
M_{11}= & U_{11} M_{11}^{(v)} U_{11}+U_{11} M_{12}^{(v)} U_{21}+\underline{U_{11} M_{13}^{(v)} U_{31}} \\
& +U_{21} M_{21}^{(v)} U_{11}+U_{21} M_{22}^{(v)} U_{21}+U_{21} M_{23}^{(v)} U_{31} \\
& +U_{31} M_{31}^{(v)} U_{11}+U_{31} M_{32}^{(v)} U_{21}+U_{31} M_{33}^{(v)} U_{31}=m_{v_{e}},
\end{aligned}
$$




$$
\begin{aligned}
M_{21}= & U_{12} M_{11}^{(v)} U_{11}+U_{12} M_{12}^{(v)} U_{21}+\underline{U_{12} M_{13}^{(v)} U_{31}} \\
& +U_{22} M_{21}^{(v)} U_{11}+U_{22} M_{22}^{(v)} U_{21}+U_{22} M_{23}^{(v)} U_{31}, \\
& +U_{32} M_{31}^{(v)} U_{11}+U_{32} M_{32}^{(v)} U_{21}+U_{32} M_{33}^{(v)} U_{31}=0,
\end{aligned}
$$

$$
\begin{aligned}
M_{31}= & U_{13} M_{11}^{(v)} U_{11}+U_{13} M_{12}^{(v)} U_{21}+\underline{U_{13} M_{13}^{(v)} U_{31}} \\
& +U_{23} M_{21}^{(v)} U_{11}+U_{23} M_{22}^{(v)} U_{21}+U_{23} M_{23}^{(v)} U_{31}, \\
& +U_{33} M_{31}^{(v)} U_{11}+U_{33} M_{32}^{(v)} U_{21}+U_{33} M_{33}^{(v)} U_{31}=0,
\end{aligned}
$$

$$
\begin{aligned}
M_{12}= & U_{11} M_{11}^{(v)} U_{12}+U_{11} M_{12}^{(v)} U_{22}+U_{11} M_{13}^{(v)} U_{32} \\
& +U_{21} M_{21}^{(v)} U_{12}+U_{21} M_{22}^{(v)} U_{22}+U_{21} M_{23}^{(v)} U_{32} \\
& +\underline{U_{31} M_{31}^{(v)} U_{12}+U_{31} M_{32}^{(v)} U_{22}+U_{31} M_{33}^{(v)} U_{32}=0,}
\end{aligned}
$$

$$
\begin{aligned}
M_{13}= & U_{11} M_{11}^{(v)} U_{13}+U_{11} M_{12}^{(v)} U_{23}+U_{11} M_{13}^{(v)} U_{33} \\
& +U_{21} M_{21}^{(v)} U_{13}+U_{21} M_{22}^{(v)} U_{23}+U_{21} M_{23}^{(v)} U_{33} \\
& +\underline{U_{31} M_{31}^{(v)} U_{13}}+U_{31} M_{32}^{(\nu)} U_{23}+U_{31} M_{33}^{(v)} U_{33}=0 .
\end{aligned}
$$

The number of conditions in (19), (20), (21), (22), and (23) is 10 . But we impose the additional condition $U_{31} M_{31}^{(v)}=$ $U_{31} M_{13}^{(v)}=$ real shown as underlined parts. We choose $\delta_{\text {PMNS }}$ as the argument of $M_{31}^{(v) *}=M_{13}^{(v) *}$. Thus, the number of conditions we impose in (19), (20), (21), (22), and (23) is 11 . Then the total number of conditions we impose in $M_{\alpha \beta}^{(v) \text { diag. }}=U_{i \alpha} M_{i j}^{(v)} U_{j \beta}$ is $18=17+1$. The total number of parameters we introduced in $M^{(v)}$ and $U$ was $27=18+9$. Imposing 18 conditions, thus, there remain nine physical parameters out of 27 . These are three neutrino masses, two Majorana phases, and $\Theta_{1}, \Theta_{2}, \Theta_{3}$, and $\delta_{\text {PMNS }}$ in the PMNS matrix. Thus, from our parametrization (10), we obtain $\delta_{\text {PMNS }}=-\left(\right.$ phase of $\left.M_{31}^{(v)}\right)$.

In fact, in the anti-SU(7) model of [15], we can show this scheme. Since we have not obtained singlet representations yet, we cannot discuss two Majorana phases. ${ }^{4}$ The nonsinglets in (9) contain three neutral heavy leptons in three 10's. These are interpreted as the $N$ 's of Fig. 2c. We worked in the bases where $M^{(d)}$ and $M^{(e)}$ are diagonal. Note that the $\mathbf{5}_{H}$ couplings are a simplified version of $\mathbf{5}_{H}$. (singlets) $[2,15]$. Namely, the VEVs of the singlets multiplied with $\mathbf{5}_{H}$ are real. Therefore, the masses of $N$ of Eq. (9), resulting from 10 10 . $\left\langle\mathbf{5}_{H}\right\rangle$, are real. Namely, the Majorana masses of $N$ in Fig. 2c are real, i.e. $\left(M^{(N)}\right)^{-1}$ does not introduce a phase in $M^{(v)}$.

In the flipped-SU(5) language, both $M^{(u)}$ and the Dirac mass in $M^{(v)}$ appear from $\mathbf{1 0} \cdot \overline{\mathbf{5}} \cdot \overline{\mathbf{5}}_{H}$. Of course, the $\overline{\mathbf{5}}_{H}$

\footnotetext{
${ }^{4}$ Singlet representations in the anti-SU(7) model will be presented in the future [?].
}

couplings imply $\overline{\mathbf{5}}_{H} \cdot$ (singlets) where some singlets contain $X^{n}$. Namely, $M^{(u)}$ results from $\mathbf{1 0}$ (containing $u$ ). $\overline{\mathbf{5}}$ (containing $\left.u^{c}\right) \cdot \overline{\mathbf{5}}_{H}$ couplings and the Dirac couplings for $M^{(v)}$ appear from $\mathbf{1 0}$ (containing $\left.N\right) \cdot \overline{\mathbf{5}}$ (containing $\left.v\right) \cdot \overline{\mathbf{5}}_{H}$. But our explicit calculation above needs only effective couplings. The leading term of the (31) element of the $u$-type quark mass matrix in the $\mathbf{Z}_{12-I}$ model [15] takes the form

$\overline{\mathbf{7}}\left(T_{3}\right)_{3} \cdot \mathbf{2 1}(U)_{1} \cdot \overline{\mathbf{7}}_{\mathrm{BEH}}\left(T_{6}\right) \cdot \mathbf{1}_{\mathrm{BEH}}\left(T_{3}\right)$

where the subscripts are the family indices and the twisted sectors are $T_{i}$ and the untwisted sector is $U$. The leading term of the (31) element of the neutrino mass matrix in the $\mathbf{Z}_{12-I}$ model [15] takes the form

$\overline{\mathbf{7}}\left(T_{3}\right)_{3} \cdot \overline{\mathbf{7}}\left(T_{3}\right)_{1} \cdot \mathbf{7}_{\mathrm{BEH}}\left(T_{3}\right) \cdot \mathbf{7}_{\mathrm{BEH}}\left(T_{3}\right) \cdot \mathbf{1}_{\mathrm{BEH}}\left(T_{3}\right) \cdot \mathbf{1}_{\mathrm{BEH}}\left(T_{9}\right)$.

Remember that we chose $\delta_{\mathrm{CKM}}$ as the argument of $M_{31}^{(u)}$ and $\delta_{\text {PMNS }}$ as the argument of $M_{31}^{(v) *}=M_{13}^{(v) *}$. In Eqs. (24) and (25), the only complex singlet is $\mathbf{1}_{\mathrm{BEH}}\left(T_{3}\right)$. Thus, we obtain $\delta_{\mathrm{PMNS}}=-\delta_{\mathrm{CKM}}$, realizing $n_{2}=-n_{1}$ of Eq. (12).

\section{Conclusion}

The preliminary value for $\delta_{\text {PMNS }}$ is large $[12,13]$, leading us to pose the theoretical question whether $\delta_{\mathrm{CKM}}= \pm \delta_{\mathrm{PMNS}}$ is satisfied or not. Thus, the relation between he quark and lepton parameters, if true, must originate from a kind of GUT relation (for the quark and lepton parameters) in a familiesunified model (to calculate the $\delta_{\mathrm{CKM}}$ and $\delta_{\mathrm{PMNS}}$ ). We presented a possibility for this relation if the weak $\mathrm{CP}$ violation is of spontaneous origin à la Froggatt and Nielsen [20] with only one complex VEV of a standard model singlet field $X$. Thus, proving this relation accurately hints a GUT, family unification, and spontaneous CP violation. In addition, an accurate determination of the Jarlskog phase in the PMNS matrix will pin down one class of the currently allowed CKM parametrizations by the relation $\delta_{\mathrm{CKM}}= \pm \delta_{\mathrm{PMNS}}$ by a topological argument. We have shown that $\delta_{\mathrm{CKM}} \simeq-\delta_{\mathrm{PMNS}}$ at the leading order if the GUT is the flipped-SU(5).

Acknowledgments We would like to thank Hyung Do Kim for useful communications. This work is supported in part by the National Research Foundation (NRF) grant funded by the Korean Government (MEST) (NRF-2015R1D1A1A01058449) and by the IBS (IBS-R017D1-2014-a00).

Open Access This article is distributed under the terms of the Creative Commons Attribution 4.0 International License (http://creativecomm ons.org/licenses/by/4.0/), which permits unrestricted use, distribution, and reproduction in any medium, provided you give appropriate credit to the original author(s) and the source, provide a link to the Creative Commons license, and indicate if changes were made. Funded by SCOAP ${ }^{3}$. 


\section{References}

1. K.A. Olive et al., Particle Data Group, Chin. Phys. C 38, 090001 (2014)

2. J.E. Kim, D.Y. Mo, and M-S. Seo, The CKM matrix from antiSU(7) unification of GUT families. Phys. Lett. B 749, 476 (2015). arXiv:1506.08984 [hep-ph]

3. C. Jarlskog, Commutator of the quark mass matrices in the standard electroweak model and a measure of maximal CP violation. Phys. Rev. Lett. 55, 1039 (1985). doi:10.1103/PhysRevLett.55.1039

4. C. Jarlskog, A basis independent formulation of the connection between quark mass matrices, $\mathrm{CP}$ violation and experiment. $\mathrm{Z}$. Phys. C 29, 491 (1985). doi:10.1007/BF01565198

5. J.E. Kim, D.Y. Mo, S. Nam, Final state interaction phases using data from CP asymmetries. J. Korean Phys. Soc. 66, 894 (2015). arXiv: 1402.2978 [hep-ph]

6. J.E. Kim, M.S. Seo, Parametrization of the CKM matrix. Phys. Rev. D 84, 037393 (2011). arXiv:1105.3304 [hep-ph]

7. M. Kobayashi, T. Maskawa, CP violation in the renormalizable theory of weak interaction. Prog. Theor. Phys. B 49, 652 (1973)

8. A. Ceccucci, Z. Ligeti, Y. Sakai, The CKM quark mixing matrix, in Ref. 1

9. N. Cabibbo, Unitary symmetry and leptonic decays. Phys. Rev. Lett. 10, 531 (1963)

10. B. Pontecorvo, Mesonium and anti-mesonium. Phys. JETP 6, 429 (1957). (Zh. Eksp. Teor. Fiz. 33, 549 (1957))

11. Z. Maki, M. Nakagawa, S. Sakata, Remarks on the unified model of elementary particles. Prog. Theor. Phys. B 28, 870 (1962)

12. Y. Oyama (for T2K Collaboration), Current status of the T2K experiment, Talk presented at Planck 2015 (Ioannina, Greece, 2015)

13. D.V. Forero, M. Tortola, J. Valle, Neutrino oscillations refitted. Phys. Rev. D 90, 093006 (2014). arXiv:1405.7540 [hep-ph]

14. H. Georgi, Towards a grand unified theory of flavor. Nucl. Phys. B 156, 126 (1979). doi:10.1016/0550-3213(79)90497-8

15. J.E. Kim, Towards unity of families: anti-SU(7) from $\mathbf{Z}_{12-I}$ orbifold compactification. JHEP 1506, 114 (2015). arXiv:1503.03104 [hep-ph]

16. F. Wilczek, A. Zee, Horizontal interaction and weak mixing angles. Phys. Rev. Lett. 424211979. doi:10.1103/PhysRevLett.42.421

17. Z.G. Berezhiani, The weak mixing angles in gauge models with horizontal symmetry: a new approach to quark and lepton masses. Phys. Lett. B 129, 99 (1983). doi:10.1016/0370-2693(83)90737-2

18. Z.G. Berezhiani, J.L. Chkareuli, Quark-leptonic families in a model with SU(5) $\times$ SU(3) symmetry. Sov. J. Nucl. Phys. 37, 618 (1983)

19. S.F. King, G.G. Ross, Fermion masses and mixing angles from SU(3) family symmetry. Phys. Lett. B 520, 243 (2001). arXiv:hep-ph/0108112

20. C.D. Froggatt, H.B. Nielsen, Hierarchy of quark masses, Cabibbo angles and CP violation. Nucl. Phys. B 147, 277 (1979). doi:10. 1016/0550-3213(79)90316-X

21. K. Choi, E.J. Chun, K. Hwang, W.Y. Song, Bimaximal neutrino mixing and small $U_{e 3}$ from Abelian flavor symmetry. Phys. Rev. D 64, 113013 (2001). arXiv:hep-ph/0107083

22. S. Pakvasa, H. Sugawara, Discrete symmetry and Cabibbo angle. Phys. Lett. B 73, 61(1978)

23. G. Segré, H.A. Weldon, Natural suppression of strong $P$ and $T$ violations and calculable mixing angles in $\mathrm{SU}(2) \times \mathrm{U}(1)$. Phys. Rev. Lett. 42, 1191 (1979)

24. P.F. Harrison, D.H. Perkins, W.G. Scott, Threefold maximal lepton mixing and the solar and atmospheric neutrino deficits. Phys. Lett. B 349, 137 (1995). doi:10.1016/0370-2693(95)00213-5

25. E. Ma, G. Rajasekaran, Softly broken $A_{4}$ symmetry for nearly degenerate neutrino masses. Phys. Rev. D 64, 113012 (2001). arXiv:hep-ph/0106291
26. G. Altarelli, F. Feruglio, C. Hagedorn, SUSY SU(5) grand unified model of tri-bimaximal mixing from $A_{4}$. JHEP 0803, 052 (2008). arXiv:0802.0090 [hep-ph]

27. H. Ishimori, S.F. King, A model of quarks with $\Delta\left(6 N^{2}\right)$ family symmetry. Phys. Lett. B 735, 33 (2014). arXiv:1403.4395 [hep$\mathrm{ph}]$

28. A. Blum, C. Hagedorn, M. Lindner, Fermion masses and mixings from dihedral flavor symmetries with preserved subgroups. Phys. Rev. D 77, 076004 (2008). arXiv:0709.3450

29. J.E. Kim, M-S. Seo, Quark and lepton mixing angles with a dodecasymmetry. JHEP 1102, 097 (2011). arXiv:1005.4684 [hep-ph]

30. L.M. Krauss, F. Wilczek, Discrete gauge symmetry in continuum theories. Phys. Rev. Lett. 62, 1221 (1989). doi:10.1103/ PhysRevLett.62.1221

31. L.E. Ibanez, G.G. Ross, Discrete gauge symmetries and the origin of baryon and lepton number conservation in supersymmetric versions of the standard model. Nucl. Phys. B 368, 3 (1992). doi:10. 1016/0550-3213(92)90195-H

32. S.M. Barr, D. Seckel, Planck scale corrections to axion models. Phys. Rev. D 46, 539(1992). doi:10.1103/PhysRevD.46.539

33. M. Kamionkowski, J. March-Russell, Planck scale physics and the Peccei-Quinn mechanism. Phys. Lett. B 282, 137 (1992). arXiv:hep-th/9202003

34. R. Holman, S.D.H. Hsu, T.W. Kephart, E.W. Kolb, R. Watkins, L.M. Widrow, Solutions to the strong CP problem in a world with gravity. Phys. Lett. B 282, 132 (1992). arXiv:hep-ph/9203206

35. B.A. Dobrescu, The strong CP problem versus Planck scale physics. Phys. Rev. D 55, 5826 (1997). arXiv:hep-ph/9609221

36. J.E. Kim, Natural Higgs-flavor-democracy solution of the $\mu$ problem of supersymmetry and the QCD axion. Phys. Rev. Lett. 111, 031801 (2013). arXiv:1303.1822 [hep-ph]

37. J.E. Kim, Abelian discrete symmetries $\mathbf{Z}_{N}$ from string orbifolds. Phys. Lett. B 726, 450 (2013). arXiv:1308.0344 [hep-th]

38. T.D. Lee, A theory of spontaneous T violation. Phys. Rev. D 8 , 1226 (1793). doi:10.1103/PhysRevD.8.1226

39. A.E. Nelson, Naturally weak CP violation. Phys. Lett. B 136, 387 (1984). doi:10.1016/0370-2693(84)92025-2

40. S.M. Barr, Solving the strong CP problem without the PecceiQuinn symmetry. Phys. Rev. Lett. 53, 329 (1984). doi:10.1103/ PhysRevLett.53.329

41. J.E. Kim, The CKM matrix with maximal $\mathrm{CP}$ violation from $\mathrm{Z}(12)$ symmetry. Phys. Lett. B 704, 360 (2011). arXiv:1109.0995 [hep$\mathrm{ph}]$

42. S.M. Barr, A new symmetry breaking pattern for $\mathrm{SO}(10)$ and proton decay. Phys. Lett. B 112, 219 (1982). doi:10.1016/ 0370-2693(82)90966-2

43. J.P. Derendinger, J.E. Kim, D.V. Nanopoulos, Anti-SU(5). Phys. Lett. B 139, 170 (1984). doi:10.1016/0370-2693(84)91238-3

44. I. Antoniadis, J.R. Ellis, J.S. Hagelin, D.V. Nanopoulos, The flipped $\mathrm{SU}(5) \times \mathrm{U}(1)$ string model revamped. Phys. Lett. B 231, 65 (1989). doi:10.1016/0370-2693(89)90115-9

45. J.E. Kim, B. Kyae, Flipped $\mathbf{S U}(5)$ from $\mathbf{Z}_{12-I}$ orbifold with Wilson line. Nucl. Phys. B 770, 47 (2007). arXiv:hep-th/0608086

46. R.D.Peccei, H.R. Quinn, Constraints imposed by CP conservation in the presence of instantons. Phys. Rev. D 16, 1791 (1977). doi:10. 1103/PhysRevD.16.1791

47. H. Baer, K.-Y. Choi, J.E. Kim, L. Roszkowski, Dark matter production in the early universe: beyond the thermal WIMP paradigm. Phys. Rep. 555, 1 (2015). arXiv:1407.0017[hep-ph]

48. J.E. Kim, The CKM matrix with maximal CP violation from $\mathbf{Z}_{12}$ symmetry. Phys. Lett. B 704, 360 (2011). arXiv:1109.0995 [hep$\mathrm{ph}]$

49. H. Georgi, A.E. Nelson, M. Shin, CP violation and Fritzsch mass matrices. Phys. Lett. B 150, 306 (1985). doi:10.1016/ 0370-2693(85)91016-0 
50. H.M. Georgi, S.L. Glashow, unity of all elementary particle forces. Phys. Rev. Lett. 32, 438 (1974). doi:10.1103/PhysRevLett.32.438

51. Z. Kakushadze, S.H.H. Tye, Three family $\mathrm{SO}(10)$ grand unification in string theory. Phys. Rev. Lett. 77, 261 (1996). arXiv:hep-th/9605221
52. L. Autonne, Sur les matrices hypohermitiennes et sur les matrices unitaires. Ann. Univ. Lyon 38, 1-77 (1915)

53. T. Takagi, On an algebraic problem related to an analytic theorem of Carathodory and Fejr and on an allied theorem of Landau. Japan. J. Math. 1, 83-93 (1925) 